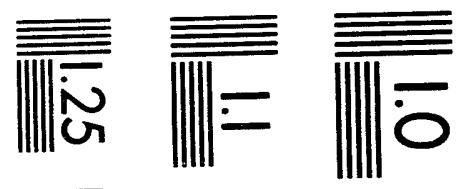

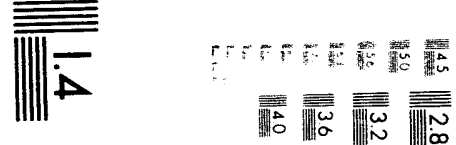

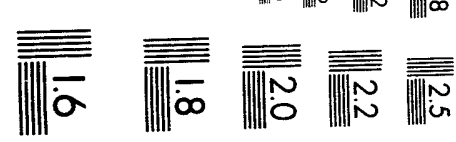



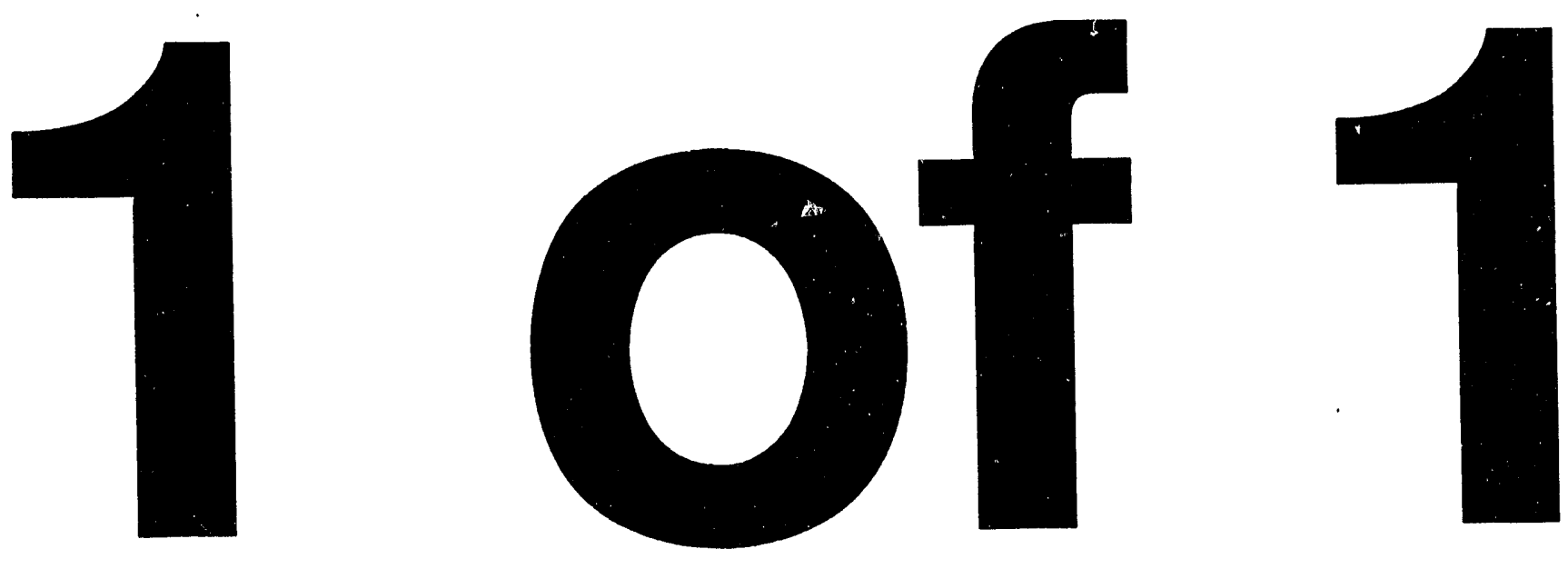


\section{INSTITUTE FOR FUSION STUDIES}

DOE/ET-53088-623

IFSR \#623-Revised

Kinctic Resonance Damping Rate of the Toroidal Ion

Temperature Gradient Mode

J.Y. Kim, Y. Kishimoto, W. Horton, and T. Tajima Institute for Fusion Studies

The University of Texas at Austin

Austin, Texas 78712

November 1993

(Revised)

THE UNIVERSITY OF TEXAS

AUSTIN 


\title{
Kinetic Resonance Damping Rate of the Toroidal Ion Temperature Gradient Mode
}

\author{
J.Y. Kim, Y. Kishimoto, W. Horton, and T. Tajima \\ Institute for Fusion Studies \\ The University of Texas at Austin \\ Austin, Texas 78712
}

\begin{abstract}
The linear damping rates of the toroidal ion temperature gradient $\left(\eta_{i}\right)$ mode due to the toroidal resonance are calculated in the local kinetic limit. The well-known Landau contour method is generalized to treat the analytic continuation problem of the guiding center dispersion function in the toroidal resonance system where the resonance occurs from both the magnetic $\nabla B$-curvature drift and the parallel ion transit drift. A detailed numerical analysis is presented for the dependence of the damping rate of the toroidal $\eta_{i}$ mode on various parameters such as $\varepsilon_{n}, k_{y}$, and the trapped electron fraction. In addition, a consideration is presented on the decay problem of the ballistic response by phase mixing in the toroidal system, which is directly related to the kinetic damping problem of the wave normal modes by the toroidal resonance.
\end{abstract}

\section{DISCLAIMER}

This report was prepared as an account of work sponsored by an agency of the United States Government. Neither the United States Government nor any agency thereof, nor any of their employees, makes any warranty, express or implied, or assumes any legal liability or responsibility for the accuracy, completeness, or usefulness of any information, apparatus, product, or process disclosed, or represents that its use would not infringe privately owned rights. Reference herein to any specific commercial product, process, or service by trade aame, trademark, manufacturer, or otherwise does not necessarily constitute or imply its endorsement, recommendation, or favoring by the United States Government or any agency thereof. The views and opinions of authors expressed herein do not necessarily state or reflect those of the United States Government or any agency thereof. 


\section{Introduction}

The ion temperature gradient mode ( $\eta_{i}$ mode) has been receiving strong interest in relation to the anomalous ion thermal transport in the high ion temperature plasmas. Numerous works have been performed to study the stability properties of the $\eta_{i}$ mode in various limits. A well-known characteristic of the $\eta_{i}$ mode is that it can be driven unstable by the two different mechanisms; the coupling of the temperature gradient with the parallel transit drift (slab mode) $)^{1-2}$ or with the toroidal magnetic $\nabla B$-curvature drift (toroidal mode).$^{3-4}$ Among these two branches, the toroidal $\eta_{i}$ mode has, in general, the larger growth rate and lower threshold value $\eta_{c}$, and thus the mode is believed to be more relevant to the anomalous ion thermal transport. Thus, most recent studies of the $\eta_{i}$ mode stability and the resulting transport are concentrating on the toroidal branch rather than the slab branch.

An important feature of the toroidal $\eta_{\mathrm{i}}$ mode is that its stability property is quite sensitive to various kinetic effects. In particular, the growth rate and real frequency of the toroidal $\eta_{i}$ mode are significantly changed when we include the kinetic effect due to the toroidal resonance. A recent attempt ${ }^{5}$ to develop the gyro-Landau fluid model equation for the toroidal $\eta_{i}$ mode reflects the importance of this toroicial resonance effect. Here, the toroidal resonance means the wave-particle interaction between the electrostatic ballooning-type mode and the guiding center drift particles in the low $\beta$ toroidal system. This toroidal resonance occurs through two different resonance mechanisms. One is the usual parallel ion transit drift resonance, as related to the ballooning structure of the mode, rather than to the shear of magnetic field as in the slab case. The other is the magnetic $\nabla B$-curvature drift resonance due to the inhomogeneity of the toroidal magnetic field. A main difference between these two resonances is that the latter occurs when the wave propagates to a particular direction, for example, the ion diamagnetic drift direction, while the former occurs for arbitrary direction of the wave propagation. 
An important effect of this toroidal resonance, besides the stabilization of the unstable toroidal $\eta_{i}$ mode, is that it gives the damping or dissipation for the stable toroidal $\eta_{i}$ mode. In the present high temperature plasma, this kinetic damping due to the resonance is expected to be the dominant dissipation mechanism for the electrostatic fluctuations, and thus might play an important role in relation to the anomalous transport. In fact, a recent fluid simulation ${ }^{6}$ of the toroidal $\eta_{i}$ mode turbulence indicates that for the reliable estimate of the saturation amplitude and the steady state transport it is quite important to know more exactly the dissipation rate of the stable long wavelength-side modes which are subject to significant resonance damping. A 3-dimensional particle simulation study ${ }^{7}$ of the toroidal $\eta_{i}$ mode turbulence also shows the importance of the resonance damping effect in relation to the calculation of the anomalous transport. In this 3D particle simulation siudy, the anomalous transport appears to follow the quasilinear diffusion model. The plasma relaxes rapidly toward a self-organized critical state ${ }^{7}$ and then a slowly oscillating steady state transport takes place near the marginal state. Here, the steady state thermal transport is closely related to the kinetic resonance response function for the toroidal dispersion relation. The more complete understanding of the toroidal resonance and the resulting damping properties may be also useful for the development of the more accurate toroidal gyro-Landau fluid model.

Motivated by these reasons, in this work we consider in more detail the resonance and damping problems of the toroidal $\eta_{i}$ mode. In the electrostatic local kinetic limit, we first consider in detail the characteristic of the toroidal resonance. Then we develop a method for the calculation of the damping rate due to this toroidal resonance. We generalize the wellknown Landau contour method ${ }^{8}$ to treat the analytic continuation problem in the toroidal resonance system which includes the magnetic $\nabla B$-curvature drift resonance as well as the parallel ion Landau resonance. This contour integral method is quite different from a previous method by Similon et al. ${ }^{9}$ where a differential equation satisfied by the dispersion function has been used to calculate the negative growth rate. While the method of Similon et al. ${ }^{9}$ 
treats well the analytic continuation problem, a difficulty appears in deriving the differential equation of the dispersion function when the dispersion function becomes a little complicated, for example, by including the $k_{\|}$or the finite Larmor radius (FLR) terms. Compared with this differential equation method, the method presented here calculates directly the dispersion function through the velocity space integral, and the analytical continuation problem is resolved by using the velocity contour which is similar to the usual Landau contour. This contour integral method appears to be more easily applicable to the more complicated forms of the dispersion function. In a particular limit with no temperature gradient and FLR terms, our method is shown in Sec. III to give the same result as that of Similon et al. ${ }^{9}$

Using the new method, we present a detailed numerical analysis of the damping rates of the toroidal $\eta_{i}$ mode. The dependences of the damping rate on various parameters such as $\varepsilon_{n}, k_{\|}$, and $k_{y}$ are numerically investigated. In addition, we consider how the trapped electrons affect the damping property of the toroidal $\eta_{i}$ mode. Finally, in the appendix we consider briefly an interesting problem closely related to the present resonant damping problem, that is, the decay problem of the ballistic response by phase mixing in the toroidal system. We show that the decay rate of the ballistic response by phase mixing is substantially slower in the toroidal system, compared with exponential decay rate in the slab system.

In Sec. II, we discuss the characteristics of the toroidal resonance. A new method to calculate the damping rate due to this toroidal resonance is then introduced in Sec. III. In Sec. IV, we present a detailed numerical analyses of the damping property of the toroidal $\eta_{i}$ mode in various parameter regimes. Finally, the conclusions are given in Sec. V.

\section{Characteristics of Toroidal Resonance}

As is well known in the local kinetic limit, the dispersion relation of the toroidal $\eta_{i}$ mode is given in dimensionless form as

$$
1+\tau=P(\omega)
$$


where $\tau=T_{i} / T_{e}$ and the dispersion function $P(\omega)$ is given by

$$
P(\omega)=\int_{-\infty}^{\infty} \frac{d v_{\|}}{\sqrt{\pi}} \int_{0}^{\infty} 2 v_{\perp} d v_{\perp} e^{-v^{2}} \frac{\omega-\omega_{*_{i}}\left(1+\eta_{i}\left(v^{2}-\frac{3}{2}\right)\right)}{\omega-\omega_{D_{i}}\left(\frac{v_{\perp}^{2}}{2}+v_{\|}^{2}\right)-\sqrt{2} k_{\|} v_{\|}} J_{0}^{2}\left(\sqrt{2} k_{\perp} v_{\perp}\right)
$$

with $\omega_{*_{i}}=k_{y}, \omega_{D i}=2 \varepsilon_{n} \omega_{*_{i}}, \varepsilon_{n}=L_{n} / R, \eta_{i}=L_{n} / L_{T_{i}}$, and $L_{n}^{-1}=-d \ln n / d r$. Here, the velocities $v_{\perp}$ and $v_{\|}$have been normalized to $\sqrt{2} v_{t i}$ where $v_{t i}=\left(T_{i} / m_{i}\right)^{1 / 2}$, with the assumption of the Maxwellian distribution. On the other hand, the frequencies $\omega$ and $\omega_{*_{i}}$ have been normalized to $v_{t i} / L_{n}$, while the wavenumbers $k_{\perp}$ and $k_{\|}$to $\rho_{i}=v_{t i} / \omega_{c i}$ and $L_{n}$, respectively. Note that under these normalizations, the positive real $\omega$ represents the ion diamagnetic direction for $k_{y}>0$.

The local kinetic dispersion relation (1) can be obtained by taking $\theta=0$ from the nonlocal gyrokinetic equation in the ballooning $\theta$ space. This local approximation implicitly assumes the strong ballooning structure of the eigenfunction and thus works well in the strong shear and short wavelength limit. In the opposite limit of weak shear or long wavelength, the eigenfunction becomes a weak ballooning structure and then the nonlocal analysis, as performed recently by Dong et al. ${ }^{10}$ and in more detail by Guo et al., ${ }^{11}$ should be considered. Guo et al. ${ }^{11}$ define the division between short and long wavelengths by $k_{y} \rho_{i}<\epsilon_{T}^{1 / 4}$ where $\epsilon_{T}=\left(L_{T_{1}} / R\right)^{1 / 4}$ from the balance of ion transit compressibility with toroidal mode growth rates for modes of width $\Delta \theta \approx 1$. One advantage of this local kinetic analysis is that it covers well the kinetic effects due to the resonance or FLR terms, compared with the nonlocal analysis which is more accurate but complicated. In this work, we study the resonance and damping property of the toroidal $\eta_{i}$ mode in this simple local kinetic limit.

Before we study the damping property of the toroidal $\eta_{i}$ mode, it is useful first to consider the important characteristics of the toroidal resonance. In Eq. (2), the toroidal resonance appears through the vanishing of the denominator $\omega-\omega_{D_{i}}\left(v_{\perp}^{2} / 2+v_{\|}^{2}\right)-\sqrt{2} k_{\|} v_{\|}=0$, which physically represents the Doppler shift of the real frequency to zero due to the magnetic $\nabla B$-curvature drift $\left(\omega_{D i}\right.$ term) and the parallel ion transit drift $\left(k_{\|}\right.$term). When $\omega$ is real. 
the denominator becomes zero along the shifted ellipse in the 2 -dimensional $\left(v_{\perp}, v_{\|}\right)$velocity space, described by

$$
\left(v_{\|}+\frac{k_{\|}}{\sqrt{2} \omega_{D i}}\right)^{2}+\frac{v_{\perp}^{2}}{2}=\frac{\omega}{\omega_{D i}}+\frac{k_{\|}^{2}}{2 \omega_{D i}^{2}}
$$

on which the strong wave-particle resonant interaction takes place. Equation (3) represents the toroidal resonance condition in the local kinetic limit, and in the following we discuss some important properties related to this condition.

Equation (3) shows that the resonance is possible only when the real frequency $\omega$ is larger than the critical $\cdots \cdot 1 e \omega_{b r}=-k_{\|}^{2} / 2 \omega_{D i}$. This critical frequency $\omega_{b r}$ is a branch point across which the analytical property of $P(\omega)$ is changed, and thus is called the branch frequency. ${ }^{9}$ The branch frequency $\omega_{b r}$ moves from $-\infty$ in the slab limit of $\omega_{D_{i}}=0$ to zero in the pure toroidal limit of $k_{\|}=0$. This behavior is related to the fact that the toroidal curvature drift moves just in one direction, while the parallel transit drift can move in both directions.

For a given $\omega_{r} \geq \omega_{b r}$ the shape of the resonant curve described by Eq. (3) changes according to the ratio between the two terms $\omega_{D i}$ and $k_{\|}$. As the ratio $k_{\|} / \omega_{D_{i}}$ increases, the radius of the resonant ellipse, which is given by $\tilde{\omega}^{1 / 2}$ for the $v_{\|}$-direction and $(2 \tilde{\omega})^{1 / 2}$ for the $v_{\perp}$-direction where $\tilde{\omega}=\left(\omega-\omega_{b r}\right) / \omega_{D_{i}}$, also increases, while the center of the ellipse, which locates at $v_{\|}=-k_{\|} / \sqrt{2} \omega_{D_{i}}$, moves to the higher velocity side along the $v_{\| \text {-axis. Here, note }}$ that the increase of the ratio $k_{\|} / \omega_{D i}$ corresponds to the mode transition from the toroidal $\left(k_{\|} / \omega_{D i} \ll 1\right)$ to the slab $\left(k_{\|} / \omega_{D i} \gg 1\right)$, even though this variation in the local limit is rather arbitrary and may not correspond to the actual transition from the toroidal to the slab branch. The elliptical shape of the resonant curve means that the resonance occurs over a range of energy $\epsilon$ values given by $\epsilon_{\min } \leq \epsilon \leq \epsilon_{\max }$. For a given $\omega_{D_{i}}, k_{\|}$, and $\omega$, the minimum and maximum resonant energies are found to be

$$
\epsilon_{\min }=\left(\sqrt{\omega-\omega_{b r}^{\prime}}-\sqrt{-\omega_{b r}}\right)^{2} / \omega_{D i}, \quad \epsilon_{\max }=2\left(\omega-2 \omega_{b r}\right) / \omega_{D i}
$$

with the pitch angle $\mu_{\min }=v_{\|} / v=1$ and $\mu_{\max }=-1 / \sqrt{1-\omega / 2 \omega_{b r}}$, respectively. Note here 
that the energies have been normalized to $m v_{t i}^{2} / 2$. The width of this resonant energy band $\left(\Delta \epsilon=\epsilon_{\max }-\epsilon_{\min }\right)$ is given by $\left(\sqrt{\omega-\omega_{b r}}+\sqrt{-\omega_{b r}}\right)^{2} / \omega_{D}$, which increases with the decreasing $\omega_{D i}$ or $\omega_{b r}$, that is, as the mode changes from the toroidal to the slab.

It is also interesting to consider how the fraction of the particles resonant with the wave in the interval $[\omega, \omega+d \omega]$ depends on the $\omega$ for given $k_{y}$ and $k_{\|}$. Assuming the Maxwellian distribution, the resonant fraction function $r(\omega)$ is given by

$$
\begin{aligned}
r(\omega) & =\int_{-\infty}^{\infty} \frac{d v_{\|}}{\sqrt{\pi}} \int_{0}^{\infty} 2 v_{\perp} d v_{\perp} e^{-v_{\perp}^{2}-v_{\|}^{2}} \delta\left(\omega-\omega_{D i}\left(v_{\perp}^{2} / 2+v_{\|}^{2}\right)-\sqrt{2} k_{\|} v_{\|}\right) \\
& =\frac{2}{\sqrt{\pi} \omega_{D i}} \int_{-\tilde{\omega}^{1 / 2}}^{\tilde{\omega}^{1 / 2}} d v_{\|} e^{-\left\{\left(v_{\|}+u\right)^{2}-2\left(v_{\|}^{2}-\tilde{\omega}\right)\right]}
\end{aligned}
$$

with $\tilde{\omega}=\left(\omega-\omega_{b r}\right) / \omega_{D_{i}}$ and $u=-k_{\|} / \sqrt{2} \omega_{D_{i}}$. Note that $r(\omega)=0$ for $\omega<\omega_{b r}$ and $\int_{-\infty}^{\infty} d \omega r(\omega)=1$. It is also easy to see that $r(\omega)$ has the dependence of $\propto(\tilde{\omega})^{1 / 2}$ near $\tilde{\omega} \sim 0$ or $\omega \sim \omega_{b r}$, while $\propto e^{-\tilde{\omega}}$ for $\tilde{\omega} \gg 1$. In Fig. 1 we show the shapes of $r(\omega)$ as a function of $\omega / \omega_{D_{i}}$ for the two values of $k_{\|} / \omega_{D_{i}}$. The function $r(\omega)$ has a steep slope near the branch frequency $\omega_{b r}$, in particular, in the pure toroidal limit of $k_{\|}=0$, and then decreases slowly with the increasing $\omega$. Compared with the pure slab case where $r(\omega)=\frac{1}{\sqrt{2 \pi\left|k_{\|}\right|}} \exp \left(-\omega^{2} / 2 k_{\|}^{2}\right)$, this shape of the toroidal resonant fraction function is quite asymmetric in terms of $\omega$. These features of $r(\omega)$ suggest that the kinetic resonance and damping characteristics will be rapidly changed near the branch frequency in the toroidal limit. Also, these features result in a different type of decay rate for the ballistic response by phase mixing in the toroidal resonant system, as is shown in appendix.

The wave-particle resonance occurring over a range of the energy band of the particles provides an interesting transport mechanism near the marginal stability state. According to the usual quasilinear fluid theory, there is no heat transport in the marginally stable or damping state even for finite amplitude fluctuations. However, a finite quasilinear heat transfer can occur from the resonant effect if the resonance occurs over. a range of particle energies. To see this, we note that the kinetic formula of the quasilinear heat flux (in the 
normalized form) is given by

$$
\mathrm{Q}(\omega)=\operatorname{Im} \int_{-\infty}^{\infty} \frac{d v_{\|}}{\sqrt{\pi}} \int_{0}^{\infty} 2 v_{\perp} d v_{\perp} e^{-v^{2}} v^{2} \frac{\omega-\omega_{* i}\left(1+\eta_{i}\left(v^{2}-\frac{3}{2}\right)\right)}{\omega-\omega_{D_{i}}\left(\frac{v_{1}^{2}}{2}+v_{\|}^{2}\right)-\sqrt{2} k_{\|} v_{\|}} J_{0}^{2}\left(\sqrt{2} k_{\perp} v_{\perp}\right)
$$

which is of the same form as the imaginary part of the P-function given in Eq. (2), but with the integrand multiplied by the energy $m v^{2} / 2$. In the marginal state, Im $P(\omega)$ becomes zero but the heat flux $Q(\omega)$ does not become zero due to the additional energy weighting in the integrand and the spread of resonant energies. Thus, a significant heat transport occurs through the resonant particles even in the marginally stable state. A recent $3 D$ particle simulation study ${ }^{7}$ of the toroidal ion temperature gradient mode turbulence transport uses this kinetic quasilinear transport model to explain the anomalous thermal transport observed in the simulation near the marginal stability state. An actual calculation of the heat transfer rate due to this kinetic effect is also given in Ref. 7 .

Finally, we note that these characteristics of the toroidal resonance can be complicated by the following two effects. One is the non-local effect, by which the branch frequency $\omega_{b r}$ will have the spatial dispersion $\Delta \omega_{b r}$ over the width of the mode so that the function $r(\omega)$ will have a smoother transition near the branch frequency. The other is the trapped electron effect. Another resonance is possible from the trapped electrons even when the real frequency is negative and thus $\omega<\omega_{b r}$. The toroidal drift wave mode will now receive the resonance over all frequency range, independently of the branch frequency. In Sec. IV, we present a detailed consideration of this trapped electron effect on the damping rate of the toroidal $\eta_{i}$ mode.

\section{Generalized Landau Contour Method}

In the previous section, we have discussed important characteristics of the toroidal resonance. From now on, we consider the damping property of the toroidal $\eta_{i}$ mode related to this toroidal resonance. It is easy to calculate the damping rate for the simple Landau resonance 
case. As will be shown, however, for the toroidal resonance case, the calculation of the damping rate is not so straightforward due to the two dimensional velocity dependence of the magnetic $\nabla B$-curvature drift term. Thus, in this section, we introduce a new method to calculate the damping rate in the toroidal resonance system.

We note first from the dispersion relation (1) that to obtain the eigenvalues of the unstable or stable modes we need just to calculate the complex dispersion function $P(\omega)$. For simplicity, from now on, we consider the following simple form of the $P$-function, instead of Eq. (2),

$$
P\left(\omega^{\prime}\right)=\int_{0}^{\infty} v_{\perp} d v_{\perp} \int_{-\infty}^{\infty} d v_{\|} \frac{f\left(\omega, v_{\perp}, v_{\|}\right)}{\omega-a v_{\perp}^{2}-b v_{\|}^{2}-c v_{\|}}
$$

where $f$ is an analytic function related to the equilibrium distribution of the particles, and $a, b, c$ are assumed to be real constants. The $P$-function is to be analytically continued from $\gamma=\operatorname{Im}(\omega)>0$ to $\gamma=\operatorname{Im}(\omega)<0$.

For the stable cases of $\gamma=\operatorname{Im}(\omega) \leq 0$, we need to carry out the analytic conilinuation of the dispersion function $P(\omega)$. A method to satisfy the analyticity condition for the stable case is to take a proper deformation of the velocity integration path into the complex plane to keep the resonant poles on the same side of the path of integration. ${ }^{8}$ The dispersion function $P(\omega)$ is then given as a sum of the residue value and the direct integration or the principal value, from the residue theorem.

The problem we encounter in this approach is that a difficulty occurs in calculating the residue value for the toroidal resonance. Unlike the Landau resonance case where the resonant pole is given as a point of $v_{\|}=\omega / c$ so that the residue can be easily calculated, in the toroidal case where the resonance condition is given as

$$
a v_{\perp}^{2}+b v_{\|}^{2}+c v_{\|}=\omega
$$

from Eq. (7), the resonant poles appear as a complex contour in the two dimensional velocity space of $v_{\perp}$ and $v_{\|}$. Thus, the toroidal resonant condition does not explicitly determine the 
values of two complex resonant poles of $v_{\perp}$ and $v_{\|}$, but gives just the relation between the two values. To explain this more explicitly, we note that the residue of the $P(\omega)$ given in Eq. (7) can be written as

$$
R(\omega)=2 \pi i \int_{C_{1}} d v_{\|} f\left[\omega, v_{\perp}\left(\omega, v_{\|}\right), v_{\|}\right]
$$

with $v_{\perp}$ given from Eq. (8) in terms of $\omega$ and $v_{\|}$. Then, we have a difficulty in choosing the complex contour $C_{1}$ in the $v_{\|}$space. Apparently, there are infinite number of forms for the complex curve $C_{1}$ and at this point it is not so clear which contour we should choose.

To find the correct contour, we first note that in the limit of $\gamma \rightarrow 0$ the curve $C_{1}$ should reduce to a well-defined real curve. Also, we note that for the function $P(\omega)$ to be an analytic function the residue also must be an analytic function. When we contemplate these two conditions, it is not so difficult to infer that the correct resonant complex contour set will take the form of

$$
v_{\perp}=\left(\omega+c^{2} / 4 b\right)^{1 / 2} x, \quad v_{\|}+c / 2 b=\left(\omega+c^{2} / 4 b\right)^{1 / 2} y
$$

with the real variables $x$ and $y$ which satisfy

$$
a x^{2}+b y^{2}=1
$$

Only for this contour set, the residue becomes an analytic function in terms of $\omega$. For the other resonant contours the residue should depend on $\operatorname{Im}(\omega)$ or $\operatorname{Re}(\omega)$, as well as $\omega$, and this means that it does not satisfy the analytic continuation condition.

While we have obtained the above contour set from a siniple consideration, it can be also derived through a more formal procedure. We first note that the simple ID Landau resonance is a particular case of the general $2 \mathrm{D}$ resonance. In the $2 \mathrm{D}$ velocity space of $v_{\perp}$ and $v_{\|}$, the resonant contour of the Landau resonance appears as a straight line, which is a particular limit of the curves for the general toroidal resonance cases. An important 
point here is that this shape of the resonant contour for the Landau resonance corresponds to a single coordinate line of the $v_{\perp}$ and $v_{\|}$velocity coordinate system. We note that the calculation of the residue is trivial for the Landau resonance case since here the resonance condition is expressed in terms of a single coordinate. In fact, if we change the coordinate system from the $\left(v_{\perp}, v_{\|}\right)$to the spherical coordinate system of $\left(v=\sqrt{v_{\perp}^{2}+v_{\|}^{2}}, \mu=v_{\|} / v\right)$, the resonance condition for the Landau case will also take the $2 \mathrm{D}$ form, making the same difficulty as the toroidal resonance case. Now, we note that from this consideration that the residue of the toroidal resonance case could be easily calculated, if we were to change the coordinate system of $v_{\perp}$ and $v_{\|}$to one in which the toroidal resonance contour appears as a straight line.

It is easy to find such a coordinate system for the toroidal resonance in Eq. (8). Taking a series of coordinate transforms of

$$
v_{\perp}^{\prime}=a^{1 / 2} v_{\perp}, \quad v_{\|}^{\prime}=b^{1 / 2}\left(v_{\|}+c / 2 b\right),
$$

and

$$
v^{\prime}=\sqrt{\left(v_{\perp}^{\prime}\right)^{2}+\left(v_{\|}^{\prime}\right)^{2}}, \quad \mu^{\prime}=v_{\|}^{\prime} / v^{\prime},
$$

we can see that in the spherical-like coordinate system of $\left(v^{\prime}, \mu^{\prime}\right)$, the resonance condition is expressed in terms of a single coordinate, as $\left(v^{\prime}\right)^{2}=\omega+c^{2} / 4 b$. Now, in terms of the new coordinate system of $\left(v^{\prime}, \mu^{\prime}\right)$, it is trivial to see that the resonant poles for the toroidal case are given as $\left(v^{\prime}\right)^{2}=\omega+c^{2} / 4 b$ and $-1 \leq \mu^{\prime} \leq 1$, in similar with that $\eta_{\|}=\omega / c$ and $0 \leq v_{\perp}<\infty$ for the simple Landau case. Inverting the above coordinate transforms, we can then obtain the corresponding resonant poles in the $v_{\perp}$ and $v_{\|}$coordinate system, which are found to be exactly the same as those described by Eqs. (10) and (11). Thus, we find that the resonant contour for the general toroidal resonance case can be easily obtained, as in the simple Landau resonance case, if we make the proper coordinate transformation.

Once the resonant contour is determined, it is easy to calculate the residue given in 
Eq. (9). If we use the resonant contour given in Eqs. (10) and (11), the residue takes the following definite integral

$$
R(\omega)=2 \pi i \sqrt{\omega+c^{2} / 4 d} \int_{-1}^{1} d y f\left[\omega, v_{\perp}(\omega, y), v_{\|}(\omega, y)\right]
$$

which can be easily calculated using the simple integration. Now, the dispersion function $P(\omega)$ for the stable case can be calculated by adding this residue $R(\omega)$ to the direct double integration value.

This new method to calculate the $P(\omega)$ in the stable regime may be considered as the direct generalization of the well-known Landau contour method to the more general toroidal resonance case. This contour integral method is quite different from the differential equation method by Similon et al. ${ }^{9}$ The present method seems to be easier to apply to the more complicated forms of the guiding center dispersion function, like that given in Eq. (2). Here, it may be worthwhile to present a comparison between these two methods for the example given in Similon et al. ${ }^{9}$ Figure 2 shows the values of $P(\omega)$ from the two methods (the dotted curve is from the Fig. 6 in the paper of Similon et al.) in the simple limit of $\eta_{i}=\omega_{*_{i}}=k_{\|}=0$, no FLR term and $\gamma / \omega_{D i}=-1$. We can see that two results agree exactly in this limit. (In Fig. 2, an arbitrary small displace of the functions plotted is made to allow the two curves to be visible on the graph.)

\section{Kinetic Damping Rates of Toroidal $\eta_{i}$ Mode}

As discussed in Sec. II, the resonance of the toroidal $\eta_{i}$ mode occurs when the real frequency is greater than a branch frequency $\omega_{b r}$. When the toroidal $\eta_{i}$ mode approaches to the marginal stability point, for example, by decreasing the $\eta_{i}$, the resonant damping will then occur only when the marginal real frequency is greater than the branch frequency. This means that by considering the marginal stability property of the toroidal $\eta_{i}$ morle we can see whether the damping occurs or not for given parameter regime. Thus, before we present the detailed 
numerical analysis of the damping rate using the method described in Sec. II, it is useful to consider property the marginal stability property of the toroidal $\eta_{i}$ mode.

Taking the approximation of $k_{\|}=0$, and $\left(v_{\perp}^{2} / 2+v_{\|}^{2}\right) \rightarrow \frac{2}{3} v^{2}$ in $\omega_{D}$ (constant energy model), it is straightforward to obtain the following marginal stability conditions of the marginal real frequency $\omega_{r}$ and the threshold $\eta_{c}{ }^{12-15}$

(i) for the resonant regime of $\omega / \omega_{D}>0$ or $2 \varepsilon_{n}(1+\tau)\left(1+b_{i}\right)>1$,

$$
\begin{aligned}
\omega_{r} / \omega_{D} & =\left(\eta_{c}-\frac{2}{3}\right) /\left(\eta_{c}-\frac{4}{3} \varepsilon_{n}\right), \\
\eta_{c} & \simeq \frac{4}{3} \varepsilon_{n}(1+\tau)\left(1+b_{i}\right),
\end{aligned}
$$

(ii) for the non-resonant regime of $\omega / \omega_{D}<0$,

$$
\eta_{c} \simeq 1-\frac{2}{3} \varepsilon_{n}(1+\tau)\left(1+b_{i}\right)
$$

near $\omega / \omega_{D} \sim 0$. Note that $\omega_{b r}=0$ in this case and we have approximately included the FLR effect using the function $\Gamma_{0}\left(b_{i}\right) \simeq 1 /\left(1+b_{i}\right)$ with $b_{i}=k_{y}^{2} \rho_{i}^{2}$.

These marginal stability conditions (14) and (15) say that the resonant damping occurs only when the value of $2 \varepsilon_{n}(1+\tau)\left(1+b_{i}\right)$ is larger than one. Thus, for a given parameter set, the resonance will occur better as $\varepsilon_{n}, \tau=T_{i} / T_{e}$, or $b_{i}$ becomes larger. The threshold value $\eta_{c}$ has the minimum value of $\eta_{c}=2 / 3$ at $2 \varepsilon_{n}(1+\tau)\left(1+b_{i}\right)=1$, that is, in the boundary between the resonant and non-resonant parameter regimes.

In Fig. 3, we show the marginal stability boundaries in the $\left[\varepsilon_{n}, \eta_{i}\right]$ space, calculated numerically from the dispersion relation Eq. (1), for the three cases of (a) $b_{i}=0, k_{\|}=0$, (b) $b_{i}=0.25, k_{\|}=0$, and $(\mathrm{c}) b_{i}=0.25, k_{\|}=0.5 \varepsilon_{n}$, with $\tau=1$. For each case, there are two types of the marginal stability boundary, that is, the resonant and the non-resonant, approximately given by Eqs. (14) and (15), respectively. Note that the non-resonant boundary has been 
determined by the condition $\omega_{r}=\omega_{b r}=-k_{\|}^{2} / 2 \omega_{D}<0$. For all of the three cases, the resonance occurs in the large $\varepsilon_{n}$ region of $\varepsilon_{n} \sim 1 /\left[2(1+\tau)\left(1+b_{i}\right)\right] \sim 0.21$, in good agreement with the $\varepsilon_{n}$ dependence given by Eqs. (14) and (15) derived from the above marginal stability analysis. An interesting feature shown in Fig. 3 is that in the resonant region of $\varepsilon_{n}>0.21$ the resonant damping occurs over a finite interval of $\eta_{i}$. When the $\eta_{i}$ decreases below the threshold value $\eta_{c}$, the real frequency approaches the branch frequency $\omega_{b r}$, making the damping stop. Figure 3 shows that the width of this damping region in the $\eta_{i}$ space increases with increasing $\varepsilon_{n}$. The width of the damping region also increases by the stabilizing effects of the FLR and $k_{\|}$terms in the resonant stability boundary region, as shown in the curves (b) and (c), respectively.

This marginal stability analysis is useful to see in what parameter regime the damping will occur, and can be easily performed without using the contour integral method developed in Sec. III. To calculate the actual damping rate of the toroidal $\eta_{i}$ mode, however, we need to solve the dispersion relation (1) using the contour integral method. From now on, we present the numerical results of damping rates obtained through this method. In Fig. 4, we first show the growth rate and real frequency as a function of $\eta_{i}$ for the three cases of $\epsilon_{n}=0.2,0.4$ and 0.8 with $k_{y} \rho_{i}=0.5, \tau=1$, and $k_{\|} L_{n}=0.1$ (so that $\omega_{b r} \simeq 0$ ). We first note that the damping at $\eta_{i}<\eta_{c}$ occurs more strongly as $\varepsilon_{n}$ increases, and the interval of the damping region in the $\eta_{i}$ space also increases with the increasing $\varepsilon_{n}$, as shown from the above marginal stability analysis. In the small $\varepsilon_{n}$ case of $\varepsilon_{n}=0.2$, there is no damping and the mode just oscillates when $\eta_{i}<\eta_{c}$. Figure $4(\mathrm{~b})$ shows clearly that this is because the real frequency $\omega_{r}$ is less than the branch frequency $\omega_{b r}=0$ when $\eta_{i}<\eta_{c}$. On the other hand, in the large $\varepsilon_{n}$ cases of $\varepsilon_{n}=0.4$ and 0.8 , a damping occurs over the interval where the real frequency $\omega_{r}$ is larger than the branch frequency $\omega_{b r}$. In these cases, the damping rate increases continuously with the decreasing $\eta_{i}$ until the real frequency reaches to the branch frequency, at which the damping mode rapidly changes to the oscillating mode. Here, we 
note that the rapid change from the damping mode to the oscillating mode is somewhat related to the steep slope of the resonant fraction function $r(\omega)$ near the branch frequency, as shown in Fig. 1.

From Fig. 4, we may conclude that near the marginal state the broadening of the density profile might be favorable for the confinement since then the stronger resonant damping of the fluctuation occurs (even though with the fixed temperature profile the broadening of the density profile increases slightly the growth rate of the unstable $\eta_{i}$ mode). However, it should be noted that the same resonance can also increase the thermal transport, through the quasilinear kinetic thermal transport mechanism, as discussed in Sec. II.

In Fig. 5 we show the dependence of the damping rate on the wavenumber $k_{y} \rho_{i}$ for the two cases of $k_{\|} L_{n}=0.1$ and 0.2 with $\eta_{i}=2.5, \tau=1$ and $\varepsilon_{n}=0.2$. In this case, we note that the branch frequency $\omega_{b r}=-k_{\|}^{2} / 2 \omega_{D i} \propto-1 / k_{y}$ decreases (becomes more negative) with the decreasing $k_{y}$. Figure 5 first shows that in the short wavelength region $\left(k_{y} \rho_{i}>1\right)$ the modes generally receive strong damping and this is because here she real frequency is almost constant with $\omega_{r} \gg \omega_{b r}$. On the other hand, in the long wavelength region, the damping occurs over some interval in the $k_{y}$ space. More damping occurs when $k_{\|} L_{n}=0.2$ than $k_{\|} L_{n}=0.1$, since the branch frequency $\omega_{b r}\left(\propto-k_{\|}^{2}\right)$ is smaller when $k_{\|} L_{n}=0.2$. The rapid transition of the damped mode to the oscillating mode in the long wavelength side occurs since the real frequency decreases faster than $\omega_{b r}$ when the $k_{y}$ decreases in these cases. However, this transition is not a general feature and in some other parameter regimes, for example, with the larger $\varepsilon_{n}$ or $\eta_{i}$, it is possible for the real frequency to decrease more slowly than the $\omega_{b r}$, making the mode damp continuously with the decreasing $k_{y}$ without changing to the oscillating mode. Here, we also note that when $k_{y}$ decreases with the fixed $k_{\|}$the toroidal magnetic curvature drift term $\omega_{D}\left(\propto k_{y}\right)$ becomes smaller than the parallel transit drift term $\left(k_{\|}\right)$, and the mode characteristic changes from the toroidal to the slab. The feature shown in the long wavelength region of Fig. 5 represents an aspect of how the 
damping rate changes with this mode transition.

In Fig. 6, we show the other aspect of the behaviour of the damping rate when the mode transition occurs through the different parameter variation. The value of $k_{\|}$is now changed with the fixed values of $k_{y}=0.5, \varepsilon_{n}=0.2$, and $\tau=1$. Figure 6 shows that when the ratio $X=k_{\|} / \omega_{D}$ between the two drift resonant terms becomes larger than one, which means the mode transition from the toroidal to the slab, rapid changes occur in the threshold value $\eta_{c}$, and in the real frequency, which were also shown in Ref. 16. Due to the rapid increase in the real frequency in the slab limit of $X \geq 1$, the damping occurs continuously without changing to the oscillating mode when $\eta_{i}$ decreases. This behaviour is found to be related to the abrupt coupling of the weakly damped $\eta_{i}$ mode with the heavily damped mode (which usually remains in the damped state even when $\eta_{i} \gg \eta_{c}$ ). This coupling to the heavily damped mode can occur (the case of Fig. 6) or not (the case of Fig. 5) when $X$ becomes larger than 1, according to the parameter regime.

Finally, we discuss the effect of the trapped electrons. In the local kinetic limit, the effect of the trapped electrons can be estimated using the following form of the non-adiabatic response function $P_{e}(\omega)$ of the electron,

$$
P_{e}(\omega)=\sqrt{2 \epsilon} \int_{0}^{\infty} 4 v^{2} \frac{d v}{\sqrt{\pi}} e^{-v^{2}} \frac{\omega-\omega_{\hbar_{e}}\left(1+\eta_{e}\left(v^{2}-\frac{3}{2}\right)\right)}{\omega-\alpha \omega_{D e} v^{2}}
$$

where $\epsilon=r / R, \omega_{*_{e}}=-\omega_{*_{i}} T_{e} / T_{i}, \omega_{D_{e}}=-\omega_{D i} T_{e} / T_{e}, \eta_{e}=L_{n} / L_{T_{e}}$ with $L_{T_{e}}=-\left(d \ln T_{e}(r) / d\right.$ $r)^{-1}$, and $\alpha \sim 1$. The dispersion relation including this trapped electron effect is obtained by adding the function $P_{e}(\omega)$ to the right side of Eq. (1) as

$$
1+\tau=P(\omega)+P_{e}(\omega)
$$

A detailed study of this type of the local kinetic dispersion relation is given in Romanelli and Briguglio ${ }^{17}$ and nonlocal theory by Ref. 11. As is well known, there are two important effects from the trapped electrons; (1) they destabilize more the toroidal $\eta_{i}$ mode; (2) 
they can generate an another instability, called the trapped electron mode, which propagates in the electron diamagnetic drift direction. When $\eta_{i}$ decreases, the growth rate of the toroidal $\eta_{i}$ mode decreases, while that of the trapped electron mode usually increases. In a restricted range of parameters these two modes couple to each other with the transition from the toroidal $\eta_{i}$ mode to the trapped electron mode when $\eta_{i}$ decreases. However, in most parameter regimes, these two modes are well separated. Here, we consider only the latter case, well-separated modes, to show how the damping property of of the toroidal $\eta_{i}$ mode is changed by the trapped electrons.

We first note that the direction of the electron magnetic $\nabla B$-curvature drift $\left(\omega_{D e}\right)$ is opposite to that of the ion $\left(\omega_{D_{i}}\right)$. Thus, another resonance can now occur from the trapped electrons when the frequency is negative, that is, the mode propagates to the electron diamagnetic drift direction. Thus, the toroidal $\eta_{i}$ mode now has resonant interactions over all of the frequency range by the ions (when $\omega>\omega_{b r}$ ) or the electrons (when $\omega<0$, note that the branch frequency of the trapped electron is zero since the average $k_{\|}$is zero for them). This means that there is now no oscillating mode when $\eta_{i}<\eta_{c}$.

In Fig. 7, we show the growth rate and real frequency as a function of $\eta_{i}$ of the eigenmodes, obtained from the dispersion relation (17) with the calculation of $P_{e}(\omega)$ performed similarly as $P(\omega)$ using the method described in Sec. III. It is shown that a new mode, that is, the trapped electron mode is generated, and the mode is well separated from the toroidal $\eta_{i}$ mode by the sign of the real frequency, Fig. 7b, and the dependence of the growth rate on the $\eta_{i}$ shown in Fig. 7a. The unstable toroidal $\eta_{i}$ mode at $\eta>\eta_{c}$ is further destabilized by the trapped electron effect. On the other hand, the oscillating toroidal $\eta_{i}$ mode at $\eta_{i}<r_{i c}$, which was possible with no trapped electrons, now becomes a damped mode. Figure 7 shows a discontinuous transition from the unstable to the damped toroidal $\eta_{i}$ mode. This discontinuity occurs because the growth rate of the $\eta_{1}$ mode is already negative at the point where the resonance by the trapped electron begins. Note that the branch frequency of the 
trapped electrons is zero so that the resonance by the trapped electrons begins when the real frequency of the toroidal $\eta_{i}$ mode becomes zero. If the growth rate is positive when the real frequency becomes zero, then the toroidal $\eta_{i}$ mode will be damped continuously with the decreasing $\eta_{i}$.

\section{Summary}

In this work we have studied the linear resonant damping problem of the toroidal $\eta_{i}$ mode in the local kinetic limit. We have first presented explanatior the important characteristics of the toroidal resonance. A practical contour deformatic 3 ral method has been developed to calculate the damping rate due to the toroidal pa. e-wave reson. se. A detailed numerical analysis is given for the damping rate of the toroidal $\eta_{i}$ mode in various parameter regimes. Summarizing some main results from this analysis, we find:

1. For the toroidal $\eta_{i}$ mode the resonant damping occurs if the real frequency is larger than the branch frequency, which is given by $\omega_{b r}=-k_{\|}^{2} / 2 \omega_{D}$ in the local kinetic limit. From the marginal stability analysis, we can easily find the parameter regime where the resonant damping will occur.

2. Approximately, the parameter regime for the resonant damping can be represented as $2 \varepsilon_{n}\left(1+T_{i} / T_{e}\right)\left(1+k_{y}^{2} \rho_{i}^{2}\right)>1$. Thus, the resonant ion damping sets in when $\varepsilon_{n}, T_{i} / T_{e}$, or $k_{y}$ becomes larger than a critical value.

3. The resonant damping of the toroidal $\eta_{i}$ mode usually occurs over a finite interval of $\eta_{i}$. This is because when $\eta_{i}$ decreases, the real frequency also decreases, becoming equal to the branch frequency where the resonance stops.

4. The trapped electrons, with their toroidal $\nabla B$-curvature drift motion in the direction opposite to the ions, make an another resonance possible in the negative real frequency 
region. The toroidal $\eta_{i}$ mode now has the resonant damping over all frequency regime by ions or electrons, and there is no oscillating mode.

Related to these results for the linear damping rate of the normal eigenmode, we have also shown that the decay rate of the ballistic response by the phase mixing is significantly slower in the toroidal system compared with the slab system. This change to a slower decay rate is related to the difference of the low-frequency resonance characteristics between two systems.

Finally, we would like to discuss the relevance of the present work for the linear damping rate to the anomalous transport problem. It is still not clear whether the anomalous transport occurs titrough the quasilinear weak turbulence or through the strong turbulence mechanism. The role of the linear damping rate appears to be significantly different between these two models. First, in the weak turbulence model the saturation of the fluctuations comes from the quasilinear relaxation of the temperature profile to near the marginally stable profile. In this case the kinetic resonant interaction plays two important roles: one is to induce the kinetic quasilinear transport near the marginal stability state as discussed in Sec. II; the other is to suppress the fluctuation through the resonant damping. In the strong turbulence case the calculations presented here must be extended to the resonances of the beat-waves with the particle distributions. This extension of the work to the toroidal beat-wave resonance will be considered in a future study. 


\section{Appendix: Phase Mixing Decay In the Toroidal Plasma System}

In this appendix, we consider an interesting problem which is closely related to the resonant damping problem in the main text. We study the decay problem of the ballistic response by the phase mixing. We show that the decay rate by the phase mixing of the ballistic response in the toroidal system is significantly weaker that in the slab system. In the toroidal system there is slow power-law decay of the ballistic perturbation rather than the exponential decay in the slab system.

As is well known, the ballistic response is the part of the perturbed distribution which carries the information about the initial perturbation. This ballistic response in phase space decays only on the time scale of collisions, but the perturbed density or current produced by this ballistic response decays rapidly in time by the phase mixing. In the slab system the perturbed density is well known that the decay by the phase mixing occurs exponentially as $\exp \left(-k_{\|}^{2} v_{t}^{2} t^{2} / 2\right)$ with $v_{t}=(T / m)^{1 / 2}$. Here, we show how this decay rate is changed by the toroidal magnetic $\nabla B$-curvature drift to a power law decay. For simplicity, we assume that the guiding center drift occurs in one direction (here the $y$ direction) perpendicular to the magnetic field with the velocity $v_{y}=v_{D}\left(\alpha v_{1}^{2} / v_{l}^{2}+v_{\|}^{2} / v_{i}^{2}\right)$ (here $\left.\alpha \sim 1 / 2\right)$, and consider the sinusoidal initial phase space perturbation of amplitude $\boldsymbol{A}$ given by

$$
f_{k}(t=0)=A F_{M}(v) \cos \left(k_{y} y+k_{\|} z\right)
$$

with the Maxwellian velocity distribution $F_{M}(v)=\exp \left(-v^{2} / 2 v_{i}^{2}\right) /\left(2 \pi v_{i}^{2}\right)^{3 / 2}$. The Lallistic response of this initial perturbation develops in time according to convection in phase space, so that $f(x, v, t)=f(x-v t, v, 0)$. It is easy to see that the time evolution of the perturbed 
density related to this ballistic response is given by

$$
\begin{aligned}
& \delta n_{k}(t)=\pi \int_{0}^{\infty} d v_{\perp}^{2} \int_{-\infty}^{\infty} d v_{\|} \frac{e^{-\frac{y^{2}}{2 v_{i}^{2}}}}{\left(2 \pi v_{i}^{2}\right)^{3 / 2}} \operatorname{Re}\left[A e^{i k_{y}\left(y-v_{D}\left(\alpha v_{1}^{2} / v_{i}^{2}+v_{i}^{2} / v_{i}^{2}\right) t\right)+i k_{\|}\left(z-v_{\|}(t)\right.}\right] \\
& =\operatorname{Re}\left[A e^{i k_{y} v+i k_{y} z} \frac{\exp \left(-\frac{k_{i}^{2} v_{i}^{2} t^{2}}{2\left(1+\omega_{D}{ }^{2}\right)}\right]}{\left(1+i \alpha \omega_{D} t\right)\left(1+i \omega_{D} t\right)^{1 / 2}}\right] \text {, }
\end{aligned}
$$

where $\omega_{D}=2 k_{y} v_{D}$. We can see that due to the complex time dependent exponent in Eq. (A4) there is a transition in the decay of the initial data at $t_{*}=1 / \omega_{D}$, that is,

$$
\delta n_{k}(t)= \begin{cases}A \cos \left(k_{y} y+k_{\|} z\right) \exp \left(-\frac{k_{1}^{2} v_{i}^{2} t^{2}}{2}\right) & \text { for } t<t_{*} \\ A \cos \left(k_{y} y+k_{\|} z+\frac{k_{y}^{2} v_{i}^{2} t}{2 \omega_{D}}\right) / \alpha\left(\omega_{D} t\right)^{3 / 2} & \text { for } t>t_{*} .\end{cases}
$$

At $t<t$ the phase mixing is dominated by the dispersion in the parallel drift and the decay occurs exponentially, but after the time $t_{*}$ the phase mixing is dominated by the dispersion in the guiding center drift and the decay occurs as a power law of $t^{-3 / 2}$, oscillating at the frequency $\omega_{b r}=-k_{\|}^{2} v_{t}^{2} / 2 \omega_{D}$, which is the branch frequency related to the guiding center drift resonance model.

This feature of the decay rate by phase mixing for $t>t_{*}$ may be understood in terms of the resonant fraction function $r(\omega)$ defined in Eq. (5). Note that the time evolution of the perturbed density by the phase mixing can be written as the Fourier transform of the resonance fraction function, as given by

$$
\delta \hat{n}_{k}(t)=\int_{-\infty}^{\infty} d \omega r(\omega) e^{-t \omega t}
$$

where $\delta n_{k}(t)=\operatorname{Re}\left[A e^{i k_{v} y+i k_{1} z} \delta \hat{n}_{k}(t)\right]$. As discussed in Sec. II, the resonant fraction $r(\omega)$ in the toroidal system is zero at $\omega<\omega_{b r}$, and thus Eq. (A6) can be written as

$$
\begin{aligned}
\delta \hat{n}_{k}(t) & =\int_{\omega_{b r}}^{\infty} d \omega r(\omega) e^{-i \omega t} \\
& =\omega_{D} e^{-i \omega_{b r} t} \int_{0}^{\infty} d \tilde{\omega} r(\tilde{\omega}) e^{-i \tilde{\omega} \omega_{D} t}
\end{aligned}
$$


with $\tilde{\omega}=\left(\omega-\omega_{b r}\right) / \omega_{D}$. Here, we note that $r(\omega)$ has a very smooth shape except near $\omega \sim \omega_{b r}$ as shown in Fig. 1. Thus, for $t>t_{*}=1 / \omega_{D}$ the large $\tilde{\omega}$ part of $r(\tilde{\omega})$ will be canceled out rapidly by the phase mixing, and the main contribution to $\delta \hat{n}_{k}(t)$ will come from the small $\tilde{\omega}$ region. Then, using that at the small $\tilde{\omega}$ region $r(\tilde{\omega})$ has the $\tilde{\omega}$ dependence of $\propto \tilde{\omega}^{1 / 2}$ we can see that for $t>t$ *

$$
\delta n_{k}(t) \simeq \frac{A \cos \left(k_{y} y+k_{\|} z-\omega_{b r} t\right)}{\alpha\left(\omega_{D} t\right)^{3 / 2}}
$$

which is the same as Eq. (A5). Thus, we find that the particular feature of the decay rate by the phase mixing of the ballistic response in the toroidal system is related to the low-frequency characteristic of the toroidal wave-particles resonance.

\section{Acknowledgments}

This work is supported by the U.S. Department of Energy under contract \#DE-FG05-80ET. 53088. 


\section{References}

1. L.I. Rudakov and R.Z. Sagdeev, Sov. Phys. Dokl. 6, 415 (1961).

2. B. Coppi, M.N. Rosenbluth, R.Z. Sagdeev, Phys. Fluids 10, 582 (1967).

3. B. Coppi and F. Pegoraro, Nucl. Fusion 17, 969 (1977).

4. W. Horton, D.-I. Choi, and W.M. Tang. Phys. Fluids 24, 1077 (1981).

5. R.E. Waltz, R.R. Dominguez, and G.W. Hammett, Phys. Fluids B 4, 3138 (1992).

6. B.G. Hong and W. Horton, Phys. Fluids B 2, 978 (1990).

i. Y. Kishimoto, T. Tajima, M.J. LeBrun, M.G. Gray, and J.Y. Kim, Ion Temperature Gradient-Driven Turbulent Transport, Eds. W. Horton, A. Wootton, and M. Wakatani, to be published by the American Institute of Physics, Dec. 1993.

8. N.A. Krall and A.W. Trivelpiece, Principles of Plasma Physics (McGraw-Hill, New York, 1973), p. 375.

9. P. Similon, J.E. Sedlak, D. Stotler, H.L. Berk, W. Horton, and D.-I. Choi, J. Comp. Phys. 54, 260 (1984).

10. J.Q. Dong, W. Horton, and J.Y. Kim, Phys. Fluids B 4, 1867 (1992).

11. S.C. Guo and F. Romanelli, Phys. Fluids B 5, 520 (1993).

12. W. Horton, B.G. Hong, and W.N. Tang, Phys. Fluids 31, 3147 (1988).

13. R.R. Dominguez and R.E. Waltz, Phys. Fluids 31, 3147 (1988).

14. F. Romanelli, Phys. Fluids B 1, 1018 (1989).

15. R.R. Dominguez and M.N. Rosenbluth, Nucl. Fusion 29, 844 (1989).

16. J.Y. Kim and W. Horton, Phys. Fluids B 3, 1167 (1991).

17. F. Romanelli and S. Briguglio, Phys. Fluids B 2, 754 (1990). 


\section{Figure Captions}

1. The resonant particle fraction as a function of $\omega / \omega_{D_{i}}$ for the two values of $k_{\|} / \omega_{D_{1}}=0$ and 1 .

2. The value of $P$-function as a function $\omega_{r} / \omega_{D_{i}}$ when $\omega_{i} / \omega_{D_{i}}=-1, \omega_{*_{i}}=\eta_{i}=k_{\|}=0$, and no FLR term where $\omega_{r}$ and $\omega_{i}$ represent the real and imaginary values of $\omega$ (the dotted line is from the Fig. 6 in the paper of Similon et al. ${ }^{9}$ )

3. The marginal stability boundaries in the $\left[\eta_{i}, \varepsilon_{n}\right]$ space for the three cases (a) $b_{i}=$ $0, k_{\|}=0$, (b) $b_{i}=0.25, k_{\|}=0$, (c) $b_{i}=0.25, k_{\|\|}=0.5 \varepsilon_{n}$, with $\tau=1$.

4. The normalized growth rate (a) and real frequency (b) as a function of $\eta_{i}$ for the three cases of $\varepsilon_{n}=0.2,0.4$ and 0.8 when $k_{y} \rho_{i}=0.5, \tau=1$, and $k_{\|}=0.1$.

5. The normalized growth rate (a) and real frequency (b) as a function of $k_{y} \rho_{i}$ for the two cases of $k_{\|}=0.1$ and 0.2 when $\eta_{i}=2.5, \varepsilon_{n}=0.2$, and $\tau=1$.

6. The normalized growth rate (a) and real frequency (b) as a function of $\eta_{i}$ for various $k_{\|}$values when $k_{y} \rho_{i}=0.5, \varepsilon_{n}=0.2$ and $\tau=1$.

7. The growth rate (a) and real frequency (b) of the toroidal $\eta_{i}$ mode and the trapped electron mode (TEM) when $\epsilon=0.1, \eta_{e}=2 ., k_{y} \rho_{i}=0.5, \varepsilon_{n}=0.2, k_{\|}=0.1$, and $\tau=1$. 


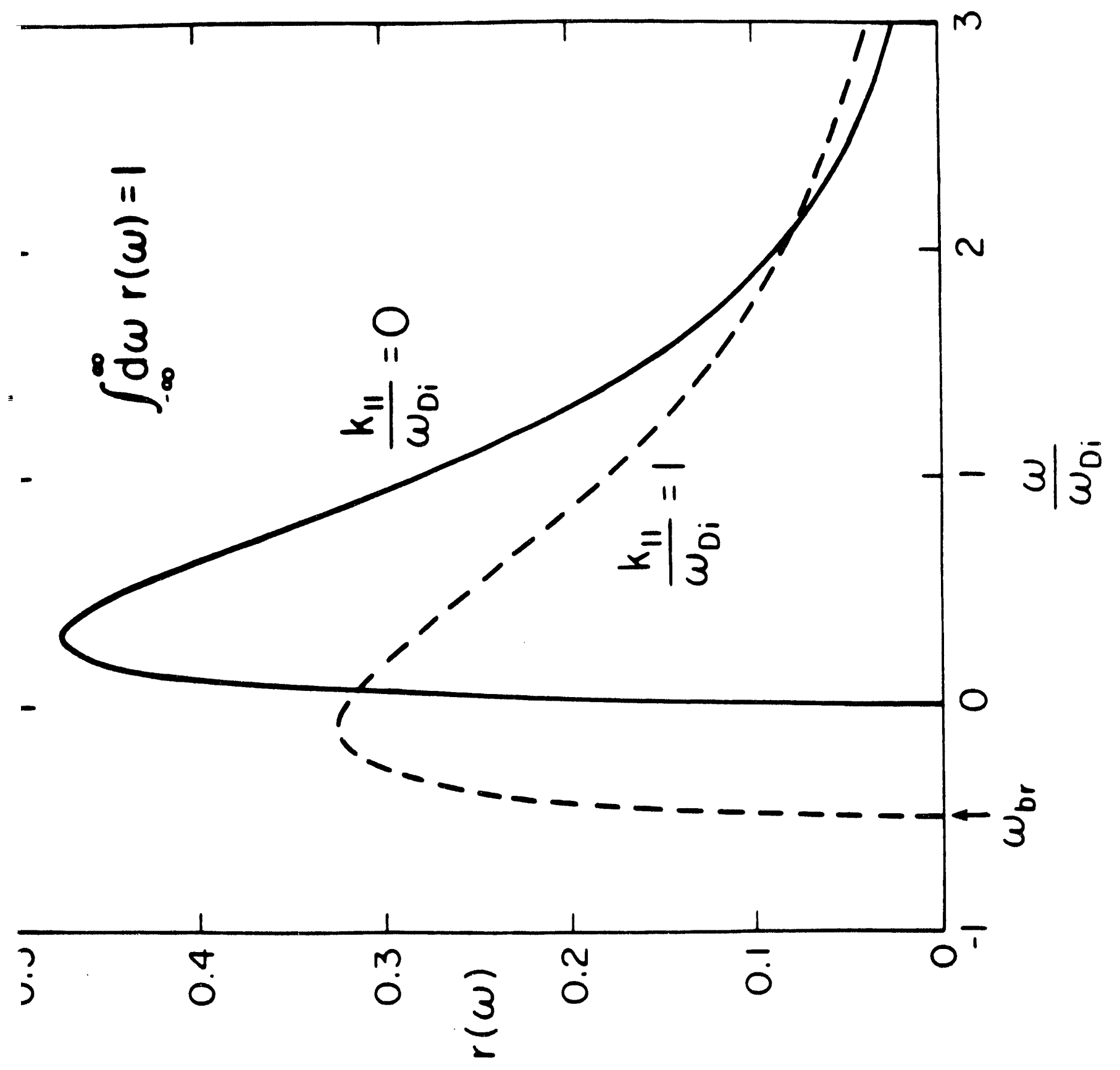

Fig. 1 


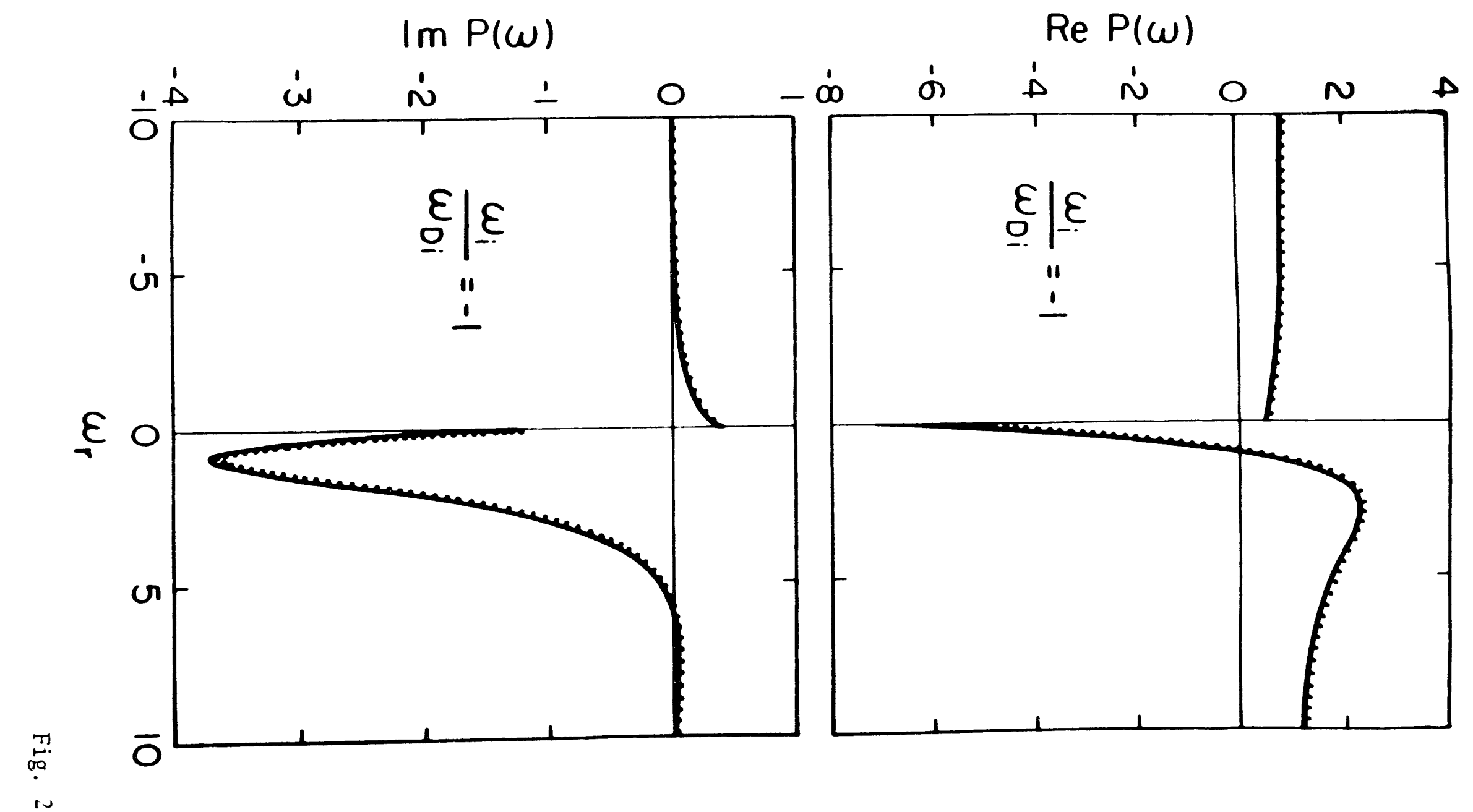




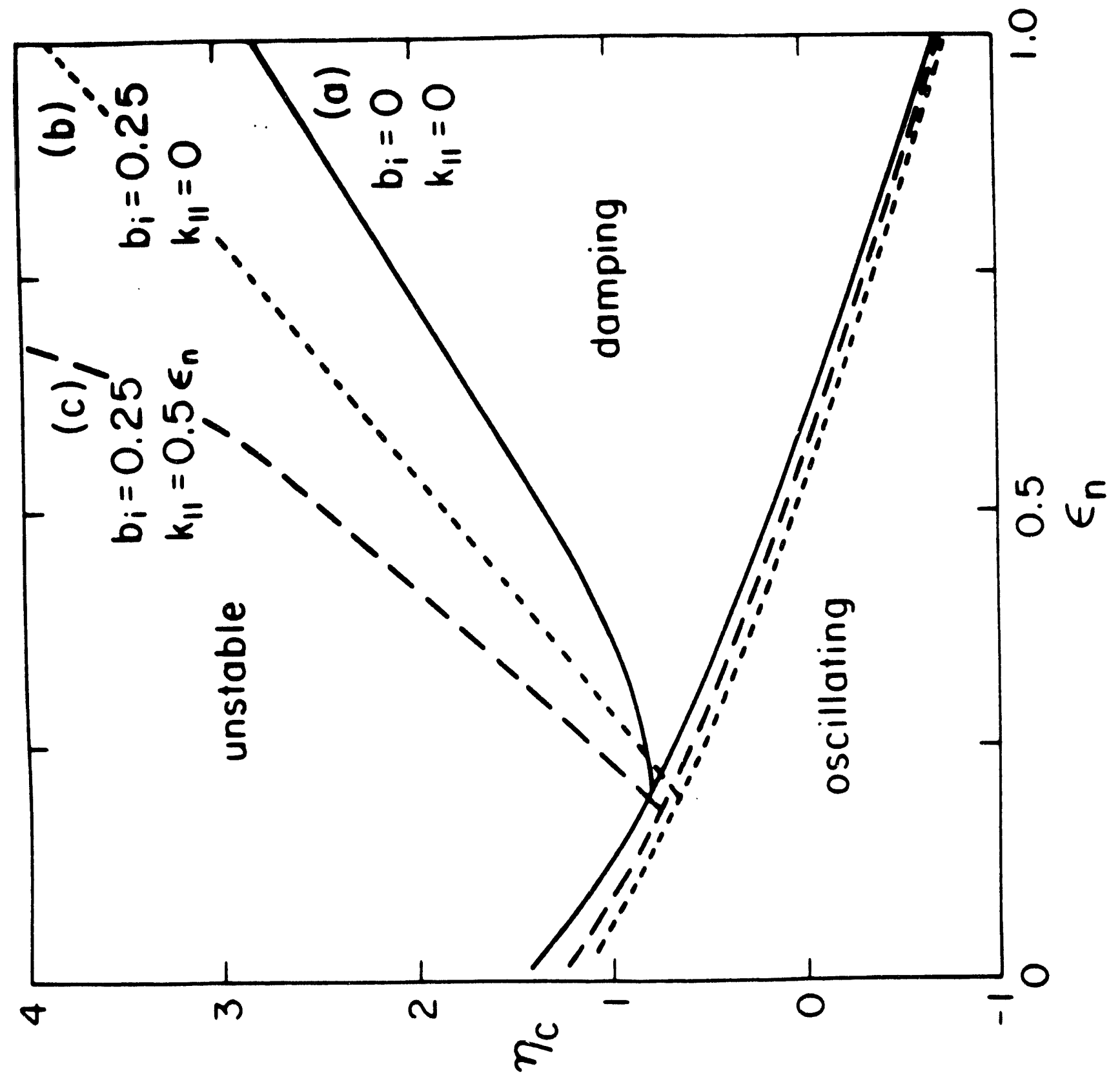

Fig. 3 


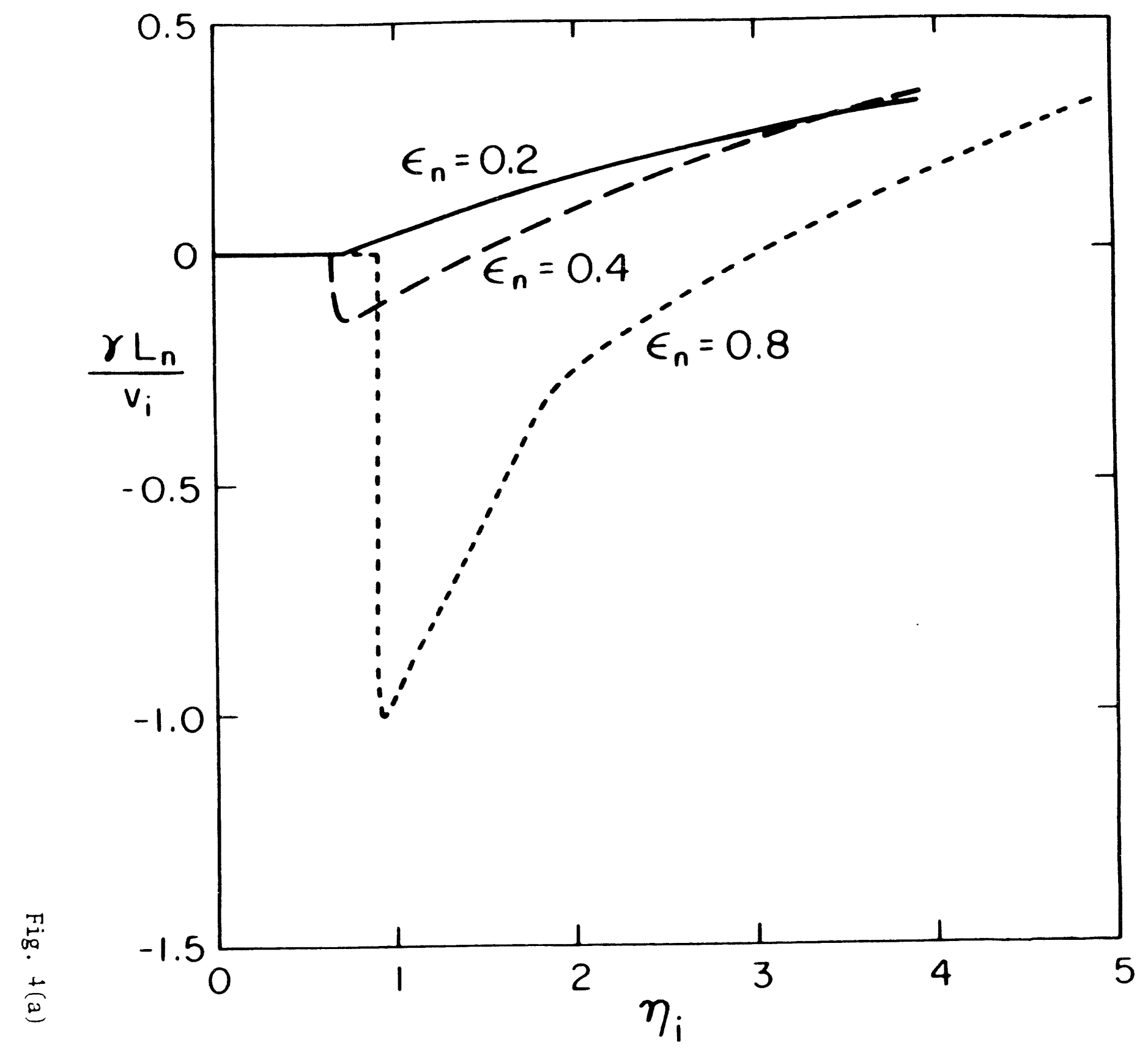




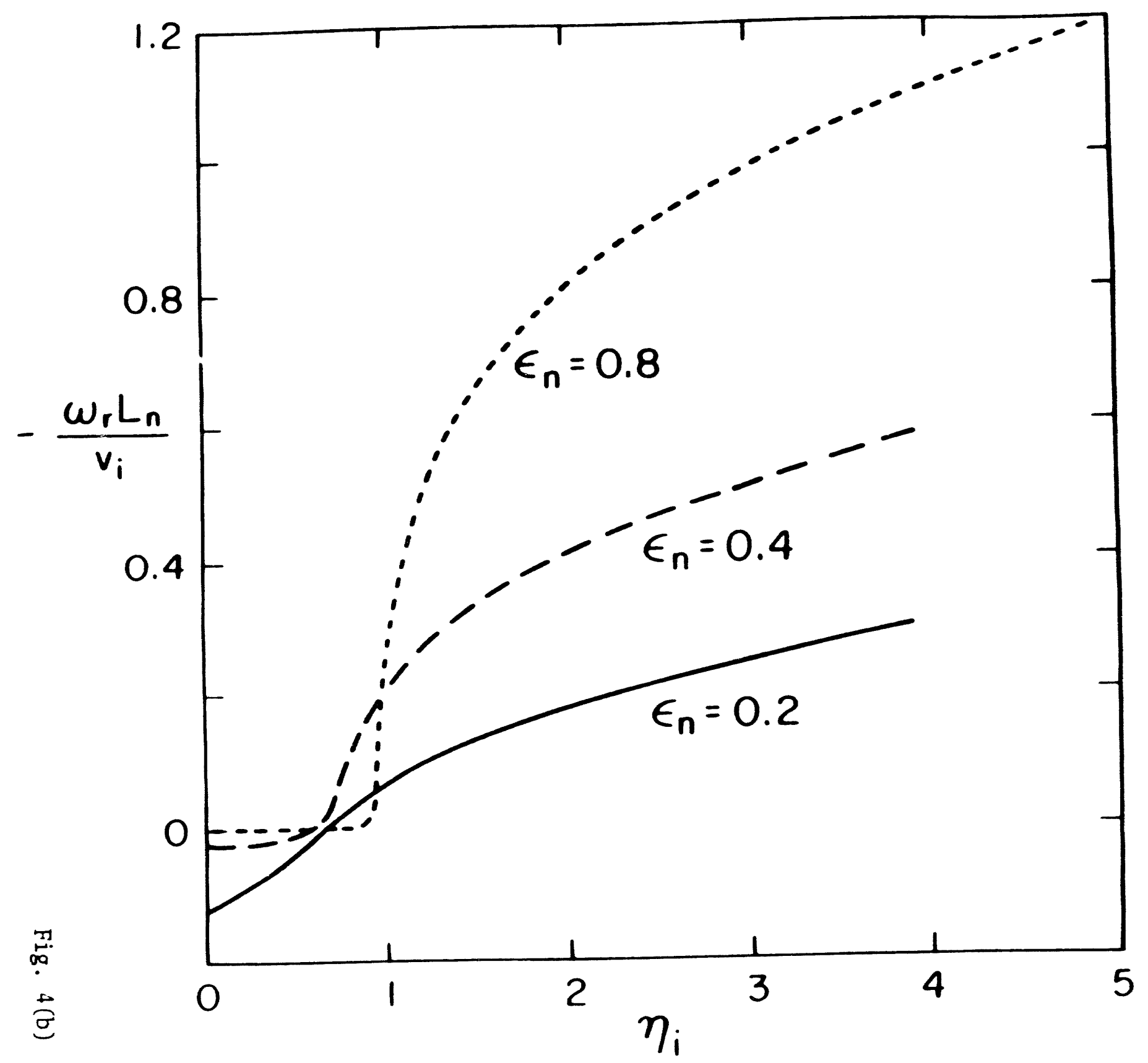




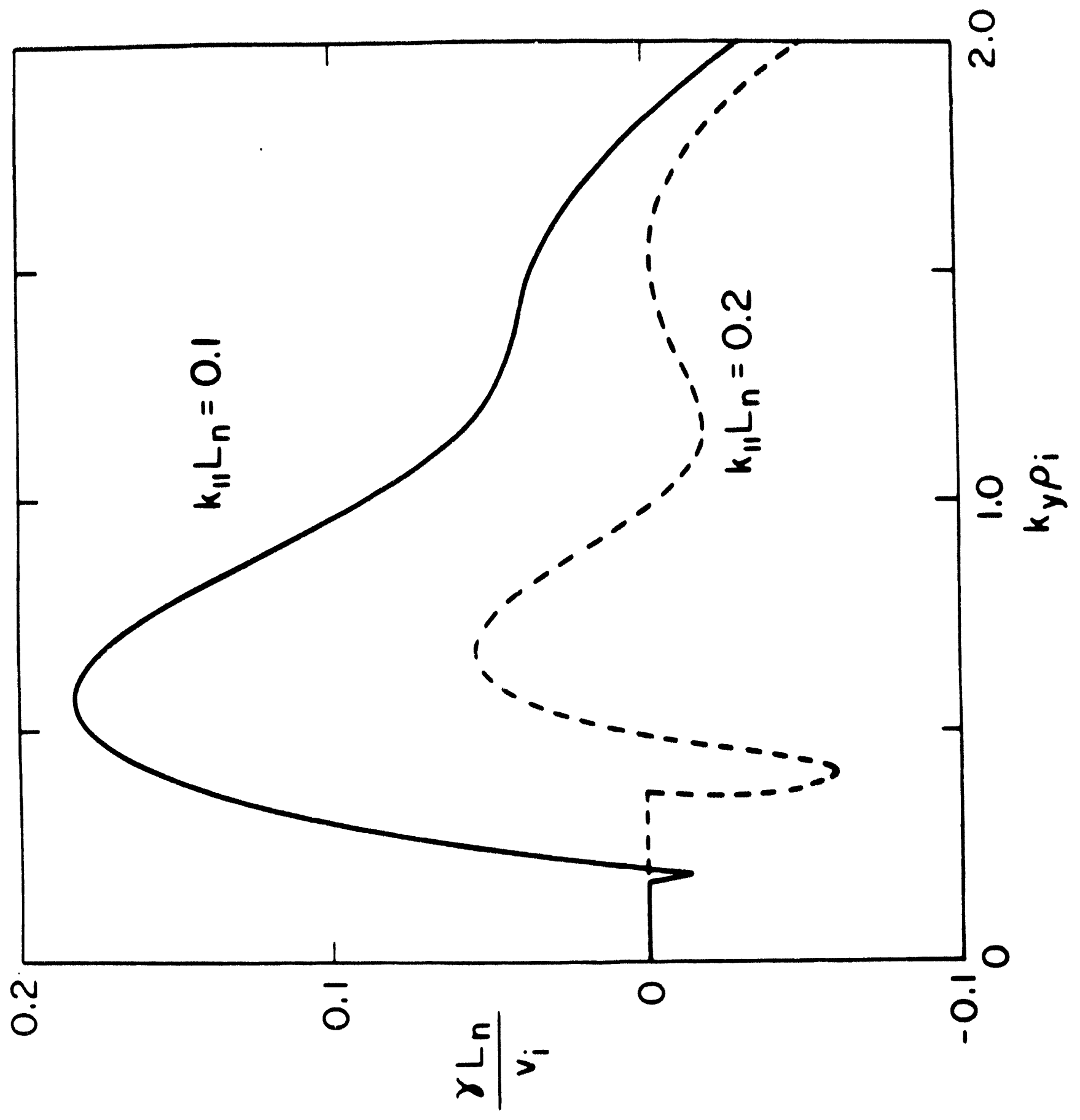

Fig. 5(a) 


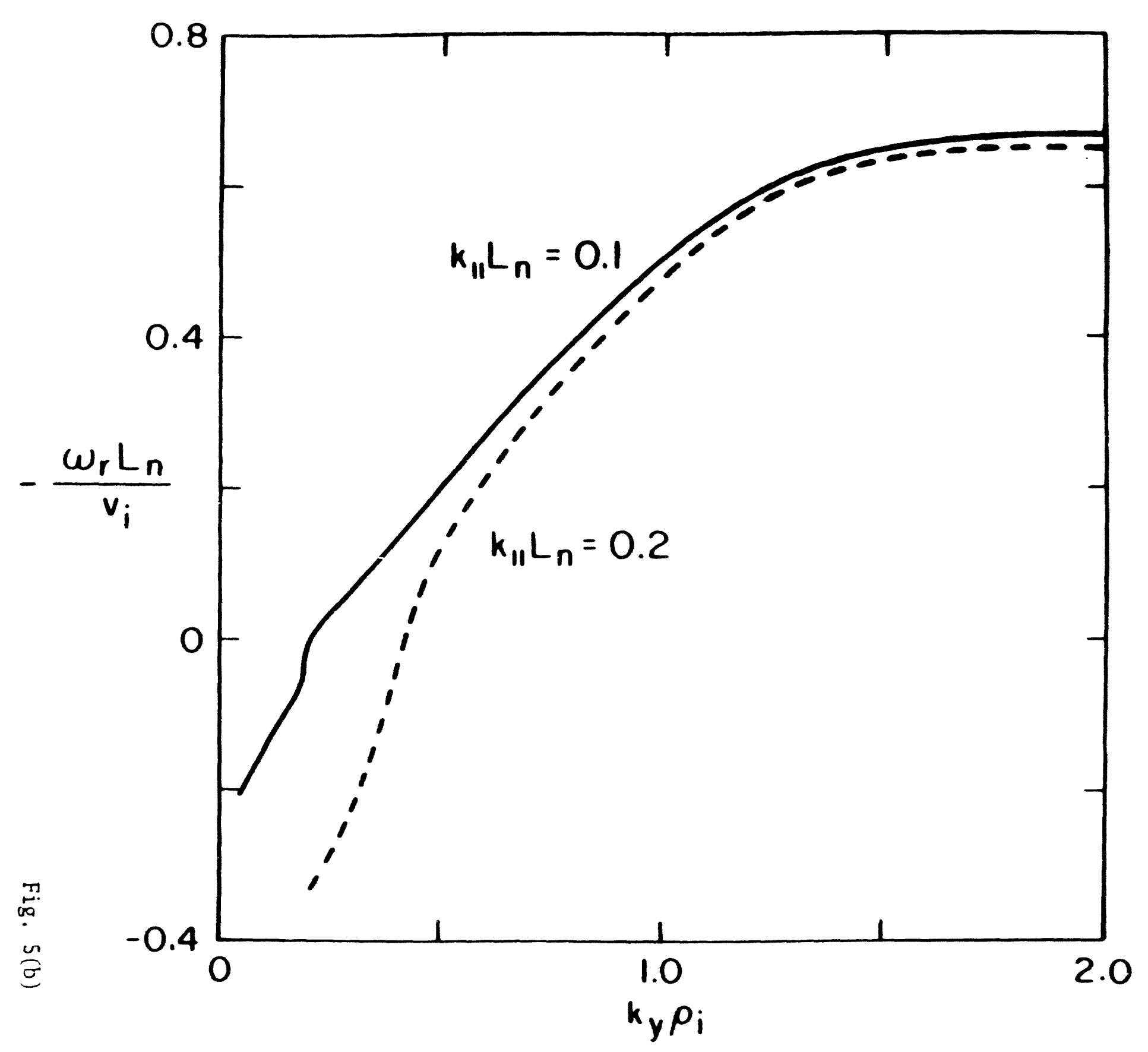




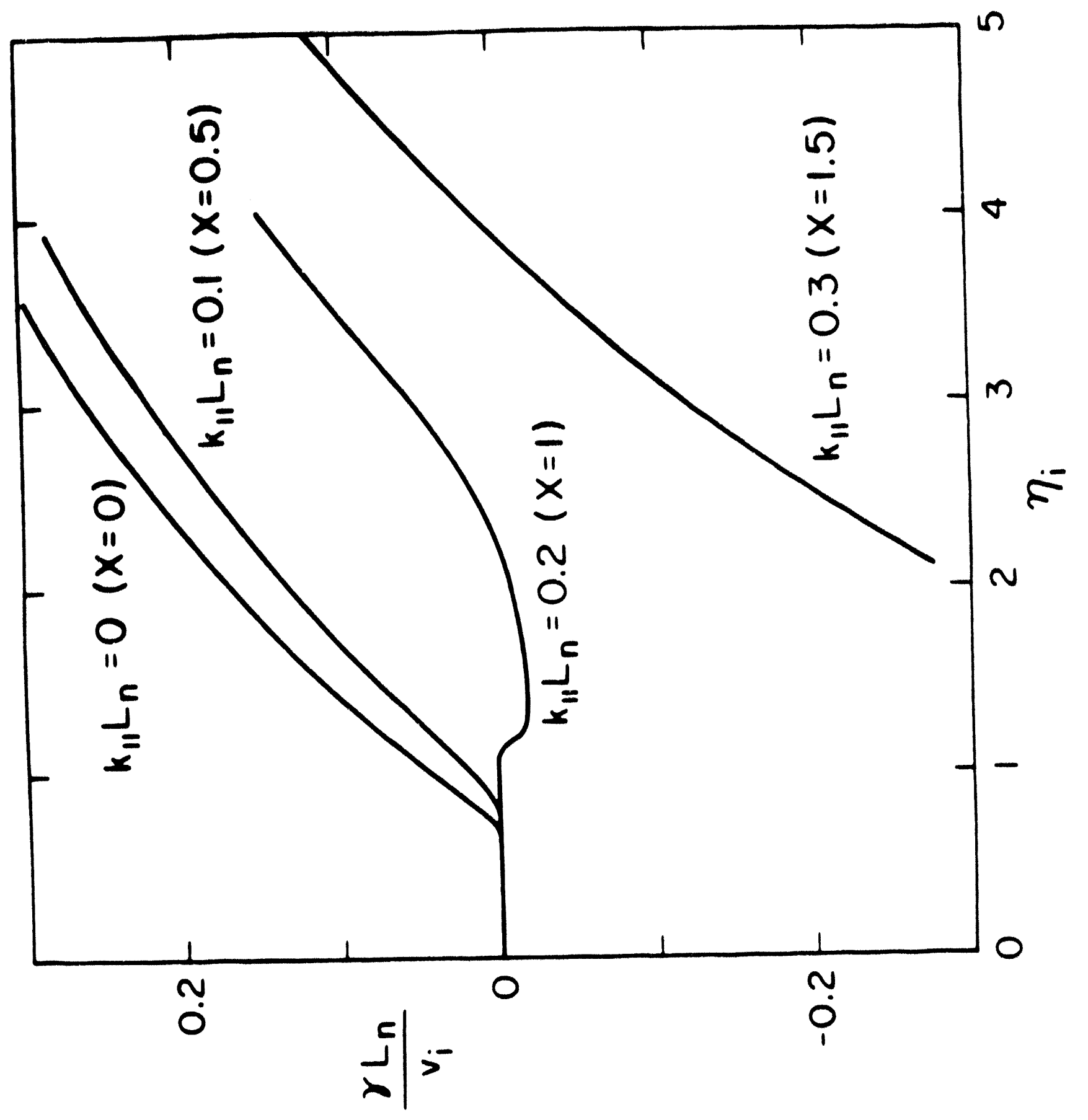

Fig. 6(a) 


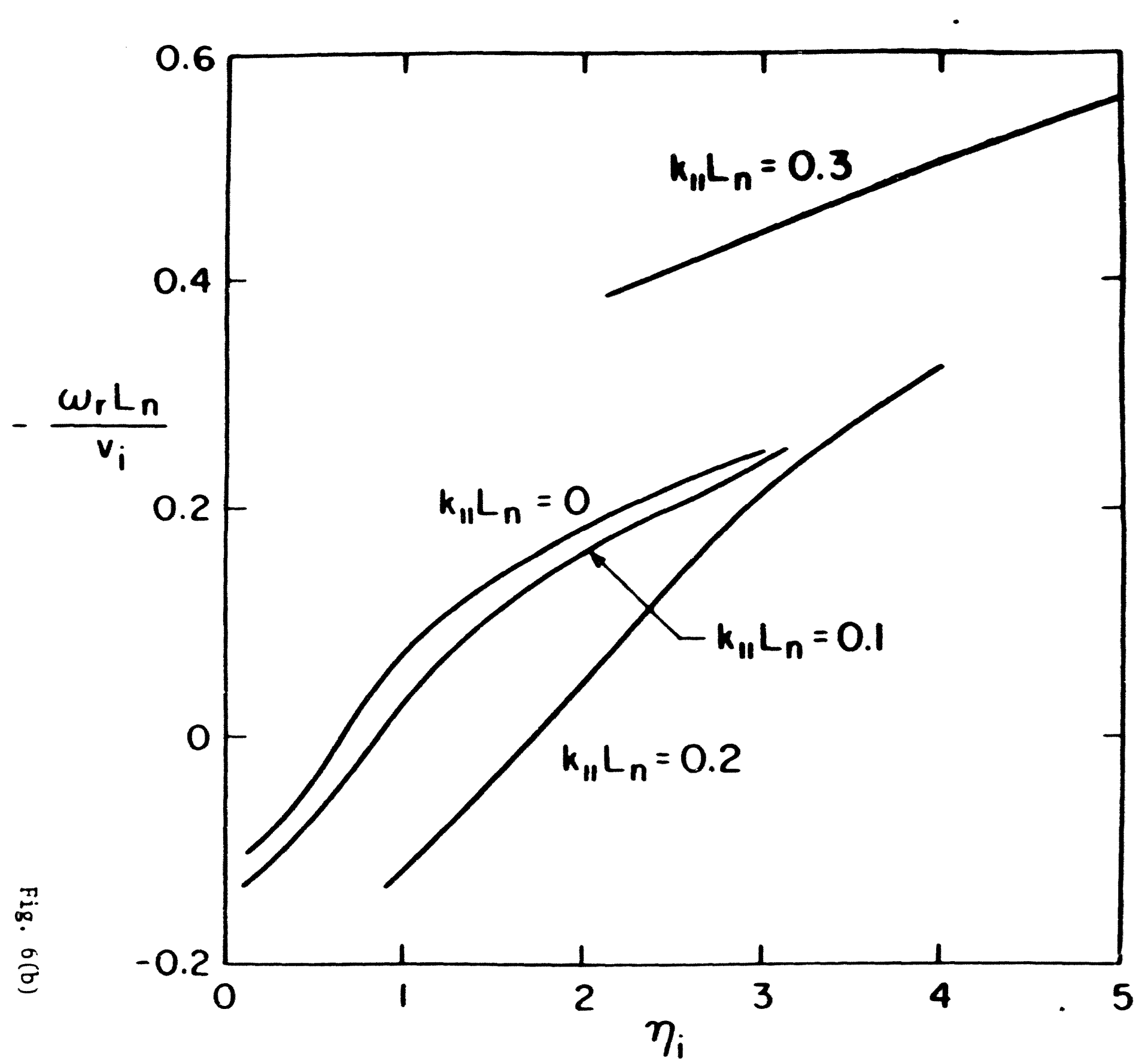




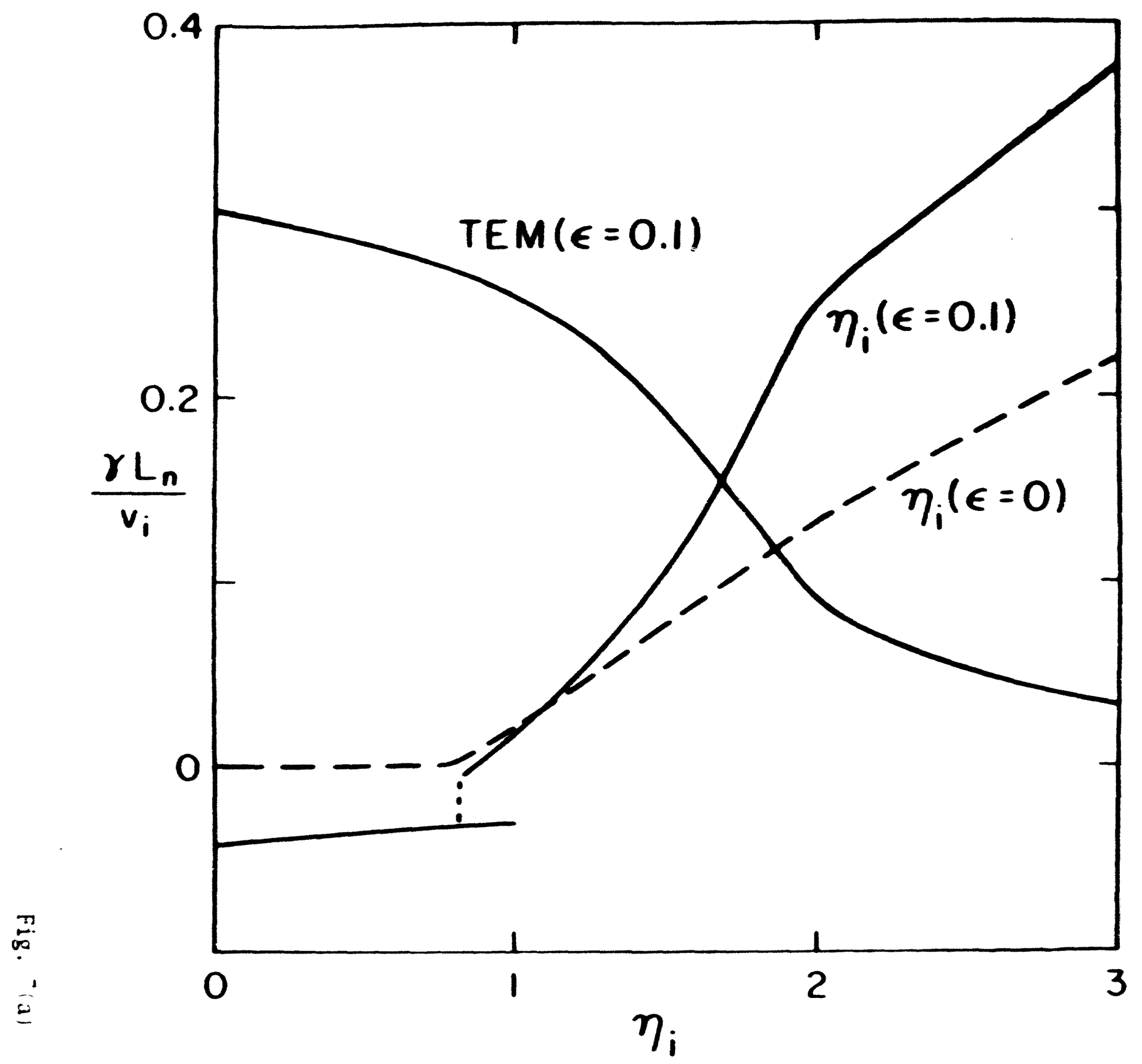




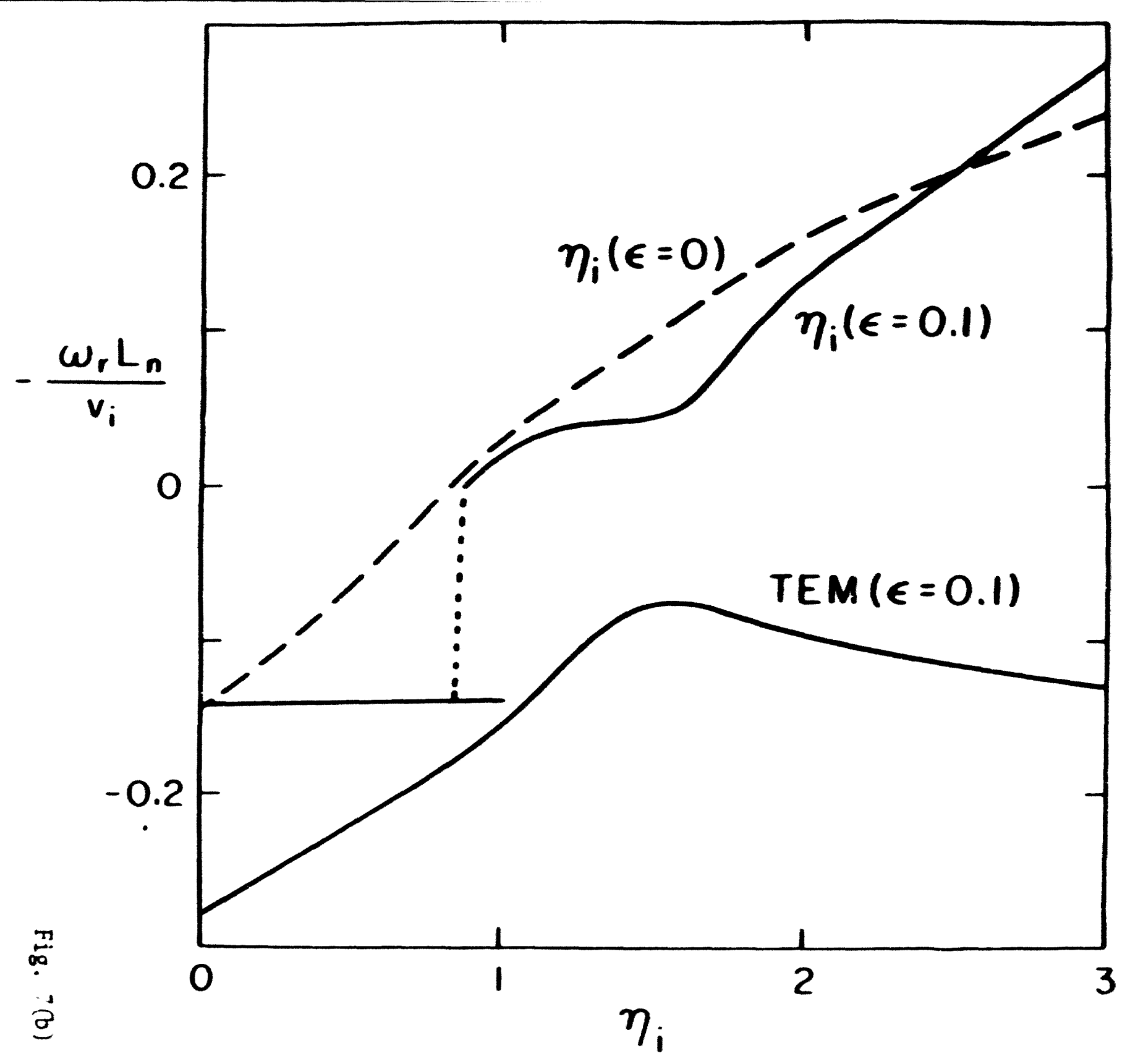



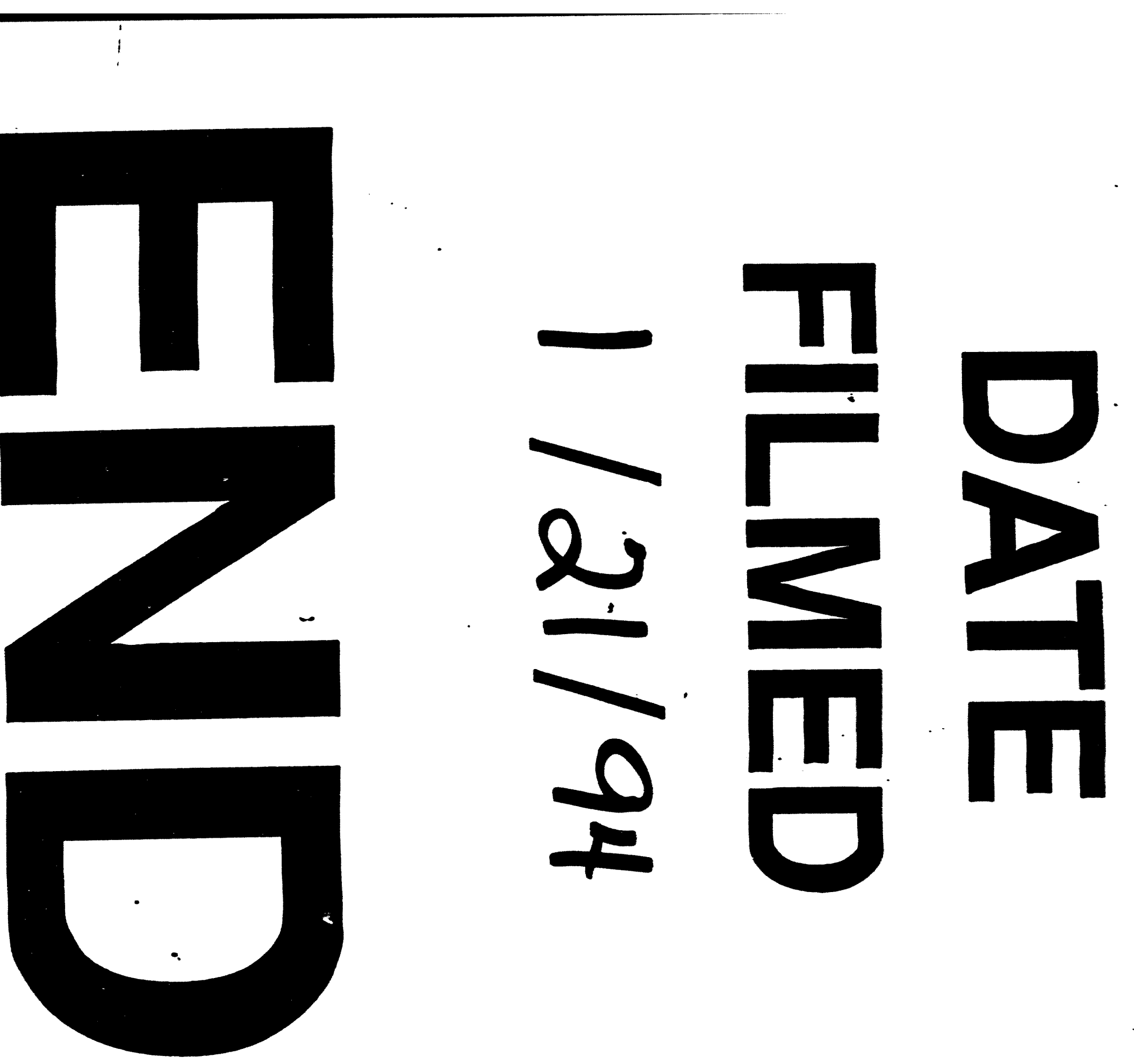
